\title{
Dealing with vesicoureteral reflux in children
}

\author{
Walid A. Farhat, MD, FRCSC
}

See related article on page 225

$\mathrm{t}$ is clear from the paper by Tamarkina and colleagues ${ }^{1}$ that as we know more about the pathophysiology of vesicoureteral reflux (VUR) in children, we know less on how to manage these patients. The pendulum is swinging toward pre-emptively treating patients with VUR, though we know that this entity is a benign condition and may not need any surgical or medical intervention. The authors raise many concerns in the treatment of VUR, the least being antibiotic prophylaxis; of note, after using the different medical approaches over past decades, flags are now being raised about the possible complications associated with antibiotics. Furthermore, we are not yet sure of the longterm outcome of deflux in the patients that we have already treated (e.g., local inflammatory changes or persistent resolution of reflux).

A critical question may arise when treating patients with VUR pre-emptively: what are we going to do for patients in whom this "incidental anesthetic" approach fails? Are we going to bring them back to the operating room, or are we going to keep them on antibiotics and just watch them?

Although there is a role for deflux in the treatment armamentarium of VUR management, it is clear that the "inci- dental anesthetic" option may be of value in some patients. For instance, boys or girls with scarred kidneys and no infections or girls who are approaching puberty may be good candidates for this approach because it is believed that these patients are at higher risk of complications later in life. On the other hand, if this option is to be part of our treatment algorithm, we need to be cognizant that most patients who don't need an ancillary surgical procedure may never be offered surgical management for their VUR.

From the Department of Surgery, University of Toronto, and the Division of Urology, Hospital for Sick Children, Toronto, Ont.

Competing interests: None declared.

\section{Reference}

1. Tamarkina E, El-Sherbiny M, Jednak R, et al. The "incidental anesthetic" — an opportunity for the endoscopic correction of vesicoureteral reflux in children. Can Urol Assoc J 2009;3:225-8.

Correspondence: Dr. Walid Farhat, Hospital for Sick Children, 555 University Ave., Toronto ON M5G 1X8; walid.farhat@sickkids.ca 